\title{
Treatment Protocols for Eating Disorders: Clinicians' Attitudes, Concerns, Adherence and Difficulties Delivering Evidence-Based Psychological Interventions
}

\author{
Glenn Waller ${ }^{1}$ \\ Published online: 18 February 2016 \\ (C) The Author(s) 2016. This article is published with open access at Springerlink.com
}

\begin{abstract}
There are several protocols in existence that guide clinicians in the implementation of effective, evidence-based psychological interventions for eating disorders. These have been made accessible in the form of treatment manuals. However, relatively few clinicians use those protocols, preferring to offer more eclectic or integrative approaches. Following a summary of the research that shows that these evidence-based approaches can be used successfully in routine clinical settings, this review considers why there is such poor uptake of these therapies in such settings. This review focuses on the role of service culture and on clinicians' own attitudes, beliefs and emotions. Possible means of enhancing uptake are considered, but these cannot be considered to be ideal solutions at present.
\end{abstract}

Keywords Eating disorders · Evidence-based practice .

Protocols $\cdot$ Manuals $\cdot$ Adherence therapist drift

\section{Introduction}

Protocols, usually codified in the form of treatment manuals, are key methods for the dissemination and reliable implementation of evidence-based psychological treatments. It is important to recognise a protocol for what it is intended to be- $-\mathrm{a}$ broad set of methods, designed to be applicable to the patient

This article is part of the Topical Collection on Eating Disorders

Glenn Waller

g.waller@sheffield.ac.uk

1 Clinical Psychology Unit, Department of Psychology, University of Sheffield, Western Bank, Sheffield S10 1NT, UK in a way that is reflective of their individual case, but guided by a set of principles. The clinical skill and artistry in the delivery of evidence-based therapy lie in how the clinician implements the protocol for the individual patient (1). However, despite this clear recognition that protocols and manuals need to be used flexibly, they are perceived negatively by many clinicians (2), who regard them as constraining their practice and artistry by limiting the individualisation of formulation and intervention approaches (1). Therapists routinely deviate from those evidence-based approaches - a phenomenon termed 'therapist drift' $(3,4)$.

Many clinicians argue that evidence-based practice is a more useful approach than the use of empirically supported interventions. Evidence-based practice combines those empirically-supported approaches (usually based on the use of a protocol/manual) with clinicians' own judgement and patients' values. However, there are two issues to consider here. First, the evidence to date does not really support the superiority of this wider 'evidence-based practice' approach. In most cases, clinician judgement results in poorer outcomes than protocol-based approaches $(5,6)$, and there is little evidence that the individualised formulations that clinicians develop are clinically reliable or useful to the patient (7). Second, in eating disorders in particular, patient values are often in direct conflict with the necessary elements of evidencebased treatments - for example, the anorexia nervosa sufferer who wants to achieve recovery (e.g. regaining their health, completing their education, being able to have children, being accepted by those around them) whilst remaining at a weight that makes this impossible, or the bulimia nervosa sufferer who wants treatment that alleviates their bulimic symptoms whilst losing weight at the same time. Therefore, one needs to be wary of the assumption that evidence-based practice (with its tripartite nature) will be superior to the single element of a protocolised evidence-based treatment. 
Clinicians working with eating disorders are potentially fortunate, in that there exists a range of evidence-based protocols that we can use to guide our clinical practice $(8-14,15$, $16,17)$. Whilst none of these achieves $100 \%$ success (see below), they have demonstrable levels of benefit and can be implemented in routine clinical practice. This review will briefly consider the evidence that we can treat the eating disorders in research and routine clinical settings, using protocolised approaches. It will then explore whether we use those approaches in routine practice and the reasons that we do or do not do so. Finally, ways in which clinicians might be encouraged to use evidence-based protocols and methods and whether such encouragement is likely to be effective will be considered.

\section{The Evidence Base for Psychological Treatments for Eating Disorders}

Once a protocol has been developed and piloted, evidencebased treatment requires two major forms of study before it can be considered to be viable. First, there need to be wellcontrolled trials of the protocol in research settings ('efficacy studies'). Then, the treatment needs to be tested in routine clinical settings ('effectiveness studies'). Both have been carried out in the field of treating eating disorders.

\section{Efficacy Studies}

There is clear evidence from large research trials that some therapies are efficacious in working with eating disorders. That evidence had been well summarised up until a few years ago, in the form of a number of reviews with very consistent conclusions (18-24). A reasonable summary of those reviews might have led to the following summary, a decade ago:

- A number of therapies had been identified that reduced the severity and presence of bulimia nervosa and binge-eating disorder, including cognitive-behavioural therapies (CBT), interpersonal psychotherapy (IPT), and dialectical behaviour therapy (DBT). Of those therapies, CBT had the strongest evidence of the greatest and most rapid effects.

- Those therapies were usually more effective if delivered in an individual, face-to-face setting.

- Medications had a limited role in the treatment of eating disorders, with some evidence for symptom alleviation in bulimia nervosa and binge-eating disorder, but no support for treating anorexia nervosa and no clear evidence for medication/psychotherapy combinations being superior. The studies were often hard to rely on as indicators of remission or recovery, as they had very short follow-ups.

- Younger adolescents with less long-lasting cases benefitted more from family-based therapies (FBT).
- Treatments for adults with anorexia nervosa had weak evidence, with weak outcomes (even when they include treatments delivered in more intensive settings), and little difference between therapies.

- There was virtually no evidence base relating to the treatment of atypical eating disorders (other than binge eating disorder), despite this category representing the largest single category of eating disorders (19).

Since those reviews were published, a number of important treatment trials have been published in the field. The key changes in our evidence base as a result can be summarised as:

- CBT has been shown to be effective in the treatment of atypical cases of eating disorder where the patient is not underweight (25), though there is no evidence that there has been any improvement in outcomes for bulimia nervosa or binge eating disorder with developments in CBT.

- Outcomes for patients with anorexia nervosa are somewhat improved, particularly for CBT $(26 \bullet \bullet, 27)$, though not in modified versions of CBT where weight gain is de-emphasised (28). CBT is now demonstrably more effective than other therapies for this group, at least by the end of therapy $(29,30)$. Its effects remain weak compared to the outcomes for patients who are not underweight, with recovery in only approximately $30 \%$ of anorexia nervosa patients entering CBT, but this is better than the outcomes demonstrated by other therapies $(15 \cdot, 30,31)$.

Other findings in recent years (32) simply reinforce the conclusions from the earlier literature, as outlined above. It is particularly noteworthy that there has been little improvement in the outcomes of pharmacotherapy in that time (33). In particular, it cannot be assumed that combining medication with psychotherapies is a useful strategy, as the combination does not reliably add to the benefits of the psychotherapies in isolation (34).

Finally, it is worth noting that the change in diagnostic criteria with DSM-5 does not appear to have altered outcome rates appreciably. However, it does mean that there is some doubt about the comparability of studies.

\section{Effectiveness}

Obviously, it is important to consider whether these evidencebased treatments can be delivered outside of highly resourced research settings. A common belief amongst clinicians is that the results from such research trials cannot be replicated in routine clinical settings, due to the patients in research settings being more carefully selected (e.g. to exclude comorbidity), an unachievable level of supervision and training for therapists, and greater resources being spent on the treatment than 
in routine practice. However, this is an empirical questioncan the results from clinical research (efficacy) be replicated in everyday practice (effectiveness)?

Fortunately, the past decade has seen the publication of several effectiveness studies that give a clear answer to this question, using large routine clinical populations of patients with different diagnoses (35-40), and the answer is clear. The clinical outcomes are very close to those found in research trials, though the attrition rate is somewhat higher. Unfortunately, the literature is almost entirely based on CBT. Therefore, there is a need for more effectiveness studies to determine whether other evidence-based therapies maintain that evidence in everyday practice. However, given the lack of support for common clinician beliefs (e.g. 'The research just does not apply here') relating to the most strongly evidenced psychological therapy, one might argue that clinicians who want to discount other therapies should be required to make the case that the evidence base cannot be applied in their place of work.

\section{How Widely Used Are the Evidence-Based Interventions?}

Despite the evidence outlined above, the simple answer to this question is: 'Not very widely'. The number of eating-disorder specialist clinicians who report adhering to evidence-based protocols and manuals is between 6 and $35 \%(41,42)$. Far more clinicians report that they use (un-tested or un-supported) mixtures of some techniques that are derived from empirically supported treatments and some techniques that are not supported even at that level $(41,43)$.

Even when clinicians say that they are using an evidencebased therapy, that claim should be treated with caution. For example, many clinicians who state that they are delivering CBT or FBT report that they omit many of the key techniques that make up those treatments $(44,45,46 \bullet, 47)$. This omission is reflected in the accounts of eating-disordered patients reporting on the treatment that they have received $(48 \cdot, 49)$, as their accounts suggest widely divergent patterns of techniques delivered under the title of CBT.

A further complication is that the same label is used for therapies with different content. CBT is a classic example of this issue, with the content differing in important ways. For example, one might not be surprised that a form of CBT for anorexia nervosa that reduced or removed the emphasis on weight gain (the key outcome variable [21]) has relatively low rates of weight gain (28). Similarly, in recent years, there has been widespread dissemination of an enhanced form of CBT (CBT-E [10]), though it has never been compared directly with the previous form of CBT to determine whether it is more effective with bulimia nervosa or binge eating disorder. Indeed, one has to be careful to understand which version of CBT-E is being used when considering outcomes, as the original two versions (broad and focused) have converged into a single version that combines the focused form with the affect regulation module of the broad form (50). Finally, studies vary in how they treat outcomes, with some studies of anorexia nervosa treating hospitalisation as a routine event and others as a reason for defining therapy as having failed $(26 \bullet \bullet, 29)$. Whilst developments and differences in delivery are clinically understandable, the key differences need to be highlighted, to ensure that differences in outcomes can be understood.

To summarise, the number of patients who receive evidence-based therapies is probably very low, outside of research settings. This pattern of therapist drift and the consequent evidence-practice gap are not confined to eating disorders $(3,4)$. However, as in other disorders, the gap is a concern because we have no idea whether it is justified in terms of patient benefits. Few clinicians document or report on the outcome of their eclectic or integrationist (or random) approaches to treatment. The use of individualised approaches to treatment of eating does not preclude measurement of effectiveness, but the lack of such measurement does not reassure others that this individual-centred approach is more effective than a protocol-based approach. Indeed, it is important to remember that an individual-centred approach based on clinician judgement has been shown to be substantially less effective than the use of a protocol-based approach (6). Therefore, once again, the obligation to demonstrate effectiveness could be argued to lie with the clinician who adopts the non-standard approach.

\section{Why Are Evidence-Based Therapies So Rarely Used for the Eating Disorders?}

Difficulties with delivering evidence-based therapies can be identified at different levels. First, there are service-centred reasons. Eating disorder services vary in their culture, and consequently, their willingness to change towards evidencebased methods. Possibly the best example of service-level reluctance is that provided by Lowe and colleagues (51), who attempted to introduce a 'normalisation of eating' module into the care package delivered by an existing set of eating disorder services. They described extreme difficulties in introducing this element of treatment, facing resistance based on the staff teams' and services' philosophy and practice of care, even though the administrators of the clinical services had approved the proposed change.

Second, patient-centred reasons exist for non-use of evidence-based methods. Patients' values can conflict with the delivery of evidence-based treatments. Many patients who have previously had therapy express a preference for an approach that is less challenging, omitting key elements such as weighing and record-keeping (52). Given the degree to which therapists omit some of the key elements of therapy (see above), it cannot be a surprise that many eatingdisordered patients have previously had several unsuccessful 
therapies and have become acculturated to the idea that therapy is a relatively unchallenging experience. In short, inadequate therapy experiences have the potential to teach patients that treatment should not be challenging in any way, when it is clear that the evidence-based approaches are based on changing ingrained behavioural, social, cognitive and emotional patterns. Of course, this avoidance of challenging is not confined to the patient, as clinicians also seem to do the same thing in order to reduce their own anxiety (see below). This joint pattern is perhaps most clearly seen when the therapist and patient fall into the pattern of discussing causes of the eating disorder without engaging in change of the maintaining factors (e.g. not changing eating patterns).

Finally, there are clinician-centred reasons for nondelivery of evidence-based approaches. One key reason applies to psychological therapies in general - many clinicians (approximately one third) are untrained in the therapy that they are employed to deliver $(53,54)$. The second important reason is the fact that many clinicians' actions typify the 'affiliation hypothesis' - because one believes in a therapy's effectiveness, it will be effective when one uses it. In the case of the eating disorders, this is a hypothesis that has been comprehensively disproved by the work of Poulsen and colleagues $\left(55^{\circ}\right)$, who have shown that therapists being affiliated to a psychodynamic approach to treating bulimia nervosa does not mean that their recovery rate comes even close to the outcome from evidence-based CBT, even when the psychodynamic therapy is several times longer.

Clinician beliefs and attitudes have a strong influence on clinical practice. Outside the field of eating disorders, it has been shown that clinicians' ratings of their skills and clinical outcomes indicate that we believe we are far more effective that we actually are (56-58). There is no reason to assume that eating disorder clinicians differ from this general pattern of beliefs. Another clinician-based factor is our knowledge of and attitudes to protocols and manuals. These vary considerably from clinician to clinician $(2,59)$, within and outside the eating disorders. Some clinicians are unaware of the existence of manuals and protocols and others hold very negative attitudes towards them. It is also clear that we hold attitudes to some elements of therapy for eating disorders that are not in keeping with the evidence about them. For example, some elements of therapy for eating disorders appear to be overvalued, such as pre-therapy motivational work $(60,61)$ and the presumed universal importance of prioritising the development of a strong working alliance in order to facilitate change $(62,63)$. In contrast, clinicians undervalue some key elements of therapy for eating disorders, such as the need to weigh patients regardless of the specific evidence-based model (64). Finally, clinicians often hold beliefs about what Meehl (5) terms 'broken leg exceptions'-leading us to exclude patients from evidence-based treatments on the grounds of apparently spurious 'justifications'. Those 'justifications' can include patient chronicity, comorbidity and complexity, even though the evidence would not support these as reasons for such an exclusion or change in practice (65-67).

Clinicians' emotions are also important in understanding the delivery of protocols and evidence-based treatment. As with other disorders $\left(68^{\bullet}\right)$, clinicians who are more anxious are less likely to deliver some of the key elements of treatment for eating disorders $(44,47)$. This avoidance of more 'demanding' elements of therapy has been suggested to be an example of clinicians engaging in their own safety behaviours - avoidance of tasks (e.g. weighing patients; changing their food intake). Such avoidance means that the clinician does not feel like a 'bad therapist' for distressing the patient, even though the longer-term result is that the patient does not improve and the clinician is less effective (4). Meehl (69) has referred to this 'protection' of patients from the demands of change as reflecting clinicians having a 'spun glass theory of the mind' of their patients - the belief that our making demands of our patients will somehow damage them (like a fragile spun-glass decoration), even though such protection means that patients cannot learn to change for the better.

\section{How Could Clinicians Be Encouraged to Use Evidence-Based Methods?}

The mean length of time for research findings to enter into routine clinical practice is a (perhaps) startling 15-20 years (70). In some cases, this delay is a matter of ignorance, and in others, it is a product of resistance at a variety of levels. Whilst ignorance of the existence of methods to treat different disorders can be overcome relatively easily through didactic methods, that is not the same as teaching or persuading clinicians to use them and to do so appropriately.

Training clinicians is a challenging area (71). Commonly, a single teaching session of a few hours to two days is treated as 'training', even though there is little evidence that this works. For example, whilst it is known that such sessions can influence knowledge of and attitudes to exposure therapy, both for eating disorders and anxiety disorders $(72,73)$, it is not yet known whether such changes translate into better use of the necessary skills in everyday practice. In short, does education translate into competence, and does competence translate into adherence to protocols and hence to more effective treatment? For example, many people have learned to drive safely whilst being closely monitored (competence), but after a short period of driving without that monitoring their driving deteriorates (non-adherence).

It is commonly assumed that clinical supervision is a means of ensuring that clinicians should adhere to best quality practice. However, there are some worrying caveats to that assumption. First, does the supervisor have an adequate grasp of the necessary evidence and skills base to be able to teach the supervisee to be adherent? Given the time it takes for evidence 
to reach practice (70) and the number of clinicians who lack training in the therapy that they deliver $(53,54,55 \bullet \cdot)$, adequate supervision is an optimistic assumption in many cases. Second, do supervisors appraise their supervisees' skills accurately? Whilst this question has not been investigated in the field of eating disorders, it has been considered elsewhere, and the worrying conclusion is that supervisors substantially overrate their supervisees' clinical skills (74), with the likely result that the therapist continues to drift off-protocol. Clearly, further research is needed to help us to understand whether or not we can trust in the value of supervision to ensure the delivery of protocolized, evidence-based therapies for eating disorders, but the evidence to date is not promising.

Of course, a strong means of persuading clinicians to change their practice should be to require them to attend to patient outcomes. The existing effectiveness studies (35-40) show that such outcomes are easily collected, and yet few services collect or respond to such information. In part, that poor response might be the product of a lack of research/audit skills or dedicated time, either of which could be rectified. However, evidence from outside the field of eating disorders (75) points to a more worrying fact- that clinicians vary substantially in their interest or willingness to attend to outcome data. If data are regarded as being irrelevant, inconvenient or challenging, then the danger is that clinicians will ignore rather than respond to them (76).

Where clinicians do attend to data, it is important that they should be encouraged to attend to the most clinically meaningful data. In the case of eating disorders, those key data appear to be those that demonstrate early change and symptom remission. There is clear evidence that behavioural and attitudinal change in the first few sessions of CBT is a critical indicator of subsequent improvement of eating disorder symptoms $(77,78,79 \bullet \bullet, 80 \bullet \bullet)$. However, whilst it is necessary, early change is not sufficient in isolation. It is also important to target some features by the end of treatment (e.g. normalisation of body image, improvement in psychosocial function), to reduce the risk of relapse (81).

\section{Conclusion}

There have been substantial advances in the treatment of eating disorders, based on the development and establishment of evidence-based approaches. Such approaches are codified in the form of manualised protocols $(8-14,15 \cdot, 16,17)$, which can be used with high levels of success in routine clinical settings (35-40). Indeed, the rate of success with protocolbased approaches is likely to be higher than that with approaches that prioritise our clinical judgement $(1,6)$.

However, this review has identified a serious gap in our use of research to drive everyday practice when working with eating disorders. Clinicians use evidence-based approaches, manuals and protocols rarely $(41-45,46 \bullet, 47,48 \bullet)$-a failure that can be attributed to our lack of knowledge, negative attitudes and emotional characteristics $(47,82)$. At present, a reliance on training and supervision to ensure competence and adherence is probably best described as an act of faith, requiring more clarity about what the training and supervision should contain. Our patients would benefit if clinicians and supervisors were to focus on clinical outcomes and the better implementation of protocols, to improve the level of patient improvement and recovery.

\section{Compliance with Ethical Standards}

Conflict of Interest The author declares that he has no conflict of interest.

Human and Animal Rights and Informed Consent This article does not contain any studies with human or animal subjects performed by any of the authors.

Open Access This article is distributed under the terms of the Creative Commons Attribution 4.0 International License (http:// creativecommons.org/licenses/by/4.0/), which permits unrestricted use, distribution, and reproduction in any medium, provided you give appropriate credit to the original author(s) and the source, provide a link to the Creative Commons license, and indicate if changes were made.

\section{References}

Papers of particular interest, published recently, have been highlighted as:

- Of importance

•• Of major importance

1. Wilson GT. Manual-based treatments: the clinical application of research findings. Behav Res Ther. 1996;34:295-314.

2. Addis ME, Krasnow AD. A national survey of practicing psychologists' attitudes towards psychotherapy treatment manuals. J Consult Clin Psychol. 2000;68:331-9.

3. Waller G. Evidence-based treatment and therapist drift. Behav Res Ther. 2009;47:119-27.

4. Waller G, Turner H. Therapist drift redux: why well-meaning clinicians fail to deliver evidence-based therapy, and how to get back on track. Behav Res Ther. 2016;77:129-37.

5. Meehl PE. Clinical versus statistical prediction: a theoretical analysis and a review of the evidence. Minneapolis: University of Minnesota Press; 1954.

6. Grove WM, Zald DH, Lebow BS, Snitz BE, Nelson C. Clinical versus mechanical prediction: a meta-analysis. Psychol Assess. 2000;12:19-30.

7. Kuyken W. Evidence-based case formulation: is the emperor clothed? In: Tarrier N, editor. Case formulation in cognitive behaviour therapy: the treatment of challenging and complex cases. London: Routledge; 2006. p. 12-35.

8. Fairburn GG, Marcus MD, Wilson GT. Cognitive-behavioral therapy for binge eating and bulimia nervosa: a comprehensive treatment manual. In: Fairburn CG, Wilson GT, editors. Binge eating: nature, assessment and treatment. New York: Guilford; 1993. p. 361-404. 
9. Gowers SG, Green L. Eating disorders: cognitive behaviour therapy with children and younger people. London: Routledge; 2009.

10. Fairburn CG. Cognitive behavior therapy and eating disorders. New York: Guilford; 2008.

11. Lock J, Le Grange D, Agras WS, Dare C. Treatment manual for anorexia nervosa: a family-based approach. New York: Guilford; 2001.

12. Le Grange D 1, Lock J. Treating bulimia in adolescents: a familybased approach. New York: Guilford; 2007.

13. Safer DL, Telch CF, Chen EY. Dialectical behavior therapy for binge eating and bulimia. New York: Guilford; 2009.

14. McIntosh VW, Jordan J, Carter FA, Luty SE, McKenzie JM, Bulik $\mathrm{CM}$, et al. Three psychotherapies for anorexia nervosa: a randomized, controlled trial. Am J Psychiatr. 2005;162:741-7.

15. Schmidt U, Magill N, Renwick B, Keyes A, Kenyon M, Dejong H, et al. The Maudsley Outpatient Study of Treatments for Anorexia Nervosa and Related Conditions (MOSAIC): comparison of the Maudsley Model of Anorexia Nervosa treatment for adults (MANTRA) with Specialist Supportive Clinical Management (SSCM) in outpatients with broadly defined anorexia nervosa: a randomized controlled trial. J Consult Clin Psychol. 2015;83:796807. A strong paper, demonstrating that more complex models and therapies do not necessarily enhance the outcomes of therapy for anorexia nervosa.

16. Waller G, Cordery H, Corstorphine E, Hinrichsen H, Lawson R, Mountford V, et al. Cognitive-behavioral therapy for the eating disorders: a comprehensive treatment guide. Cambridge: Cambridge University Press; 2007.

17. Wonderlich SA, Peterson CB, Crosby RD, Smith TL, Klein MH, Mitchell JE, et al. A randomized controlled comparison of integrative cognitive-affective therapy (ICAT) and enhanced cognitivebehavioral therapy (CBT-E) for bulimia nervosa. Psychol Med. 2014;44:543-53.

18. National Institute for Clinical Excellence. Eating disorders: core interventions in the treatment and management of anorexia nervosa, bulimia nervosa and related eating disorders (Clinical Guideline 9). London: National Collaborating Centre for Mental Health; 2004.

19. Fairburn CG, Harrison PJ. Eating disorders. Lancet. 2003;361: 407-16.

20. Hay P, Bacaltchuk J, Claudino A, Ben-Tovim D, Yong PY. Individual psychotherapy in the outpatient treatment of adults with anorexia nervosa. Cochrane Database Syst Rev. 2003;4: CD003909.

21. Bulik CM, Berkman ND, Brownley KA, Sedway JA, Lohr KN. Anorexia nervosa treatment: a systematic review of randomised controlled trials. Int J Eat Disord. 2007;40:310-20.

22. Shapiro JR, Berkman ND, Brownley KA, Sedway JA, Lohr KN, Bulik CM. Bulimia nervosa treatment: a systematic review of randomized controlled trials. Int J Eat Disord. 2007;40:321-36.

23. Brownley KA, Berkman ND, Sedway JA, Lohr KN, Bulik CM. Binge eating disorder treatment: a systematic review of randomized controlled trials. Int J Eat Disord. 2007;40:337-48.

24. Watson HJ, Bulik CM. Update on the treatment of anorexia nervosa: review of clinical trials, practice guidelines and emerging interventions. Psychol Med. 2013;43:2477-500.

25. Fairburn CG, Cooper Z, Doll HA, O'Connor ME, Bohn K, Hawker $\mathrm{DM}$, et al. Transdiagnostic cognitive-behavioural therapy for patients with eating disorders: a two-site trial with 60 week followup. Am J Psychiatr. 2009;166:311-9.

26.• Fairburn CG, Cooper Z, Doll HA, O’Connor ME, Palmer RL, Dalle Grave R. Enhanced cognitive behavior therapy for adults with anorexia nervosa: a UK-Italy study. Behav Res Ther. 2013;51:R2-8. A key study, demonstrating the potential for CBT as a first line treatment for anorexia nervosa.
27. Dalle Grave R, Calugi S, Conti M, Doll H, Fairburn CG. Inpatient cognitive behaviour therapy for anorexia nervosa: a randomized controlled trial. Psychother Psychosom. 2013;82:390-8.

28. Touyz SW, Le Grange D, Lacey H, Hay P, Smith R, Maguire S, et al. Treating severe and enduring anorexia nervosa: a randomized controlled trial. Psychol Med. 2013;43:2501-11.

29. Zipfel S, Wild B, Gross G, Friederich HC, Teufel M, Schellberg D, et al. Focal psychodynamic therapy, cognitive behaviour therapy, and optimised treatment as usual in outpatients with anorexia nervosa (ANTOP study): randomised controlled trial. Lancet. 2014;383(9912):127-37.

30. Byrne S. Principal outcomes of the Strong Without Anorexia Nervosa (SWAN) study: A multicentre randomised controlled trial of three psychological treatments for anorexia nervosa. Paper presented at the Eating Disorders Research Society Meeting; 2015, Taormina, September.

31. McIntosh VVW, Carter FA, Bulik CM, Frampton CMA, Joyce PR. Five-year outcome of cognitive behavioural therapy and exposure with response prevention for bulimia nervosa. Psychol Med. 2011;41:1061-71.

32. Lock J, Le Grange D, Agras WS, Moye A, Bryson SW, Jo B. Randomized clinical trial comparing family-based treatment with adolescent-focused individual therapy for adolescents with anorexia nervosa. Arch Gen Psychiatry. 2010;67:1025-32.

33. Crow SJ, Mitchell JE, Roerig JR, Steffen K. What potential role is there for medication treatment in anorexia nervosa? Int $\mathrm{J}$ Eat Disord. 2009;42:1-8.

34. Reas DL, Grilo CM. Current and emerging drug treatments for binge eating disorder. Expert Opinion Emerging Drugs. 2014;19: 99-142.

35. Byrne SM, Fursland A, Allen KL, Watson H. The effectiveness of enhanced cognitive behavioural therapy for eating disorders: an open trial. Behav Res Ther. 2011;49:219-26.

36. Ghaderi A. Does individualization matter? A randomized trial of standardized (focused) versus individualized (broad) cognitive behavior therapy for bulimia nervosa. Behav Res Ther. 2006;44:273-88.

37. Knott S, Woodward D, Hoefkens A, Limbert C. Cognitive behaviour therapy for bulimia nervosa and eating disorders not otherwise specified: translation from randomized controlled trial to a clinical setting. Behav Cognitive Psychother. 2015;43:641-54.

38. Peterson CB, Miller KB, Willer MG, Ziesmer J, Durkin N, Arikian A, et al. Cognitive-behavioral therapy for subthreshold bulimia nervosa: a case series. Eat Weight Disord. 2011;16: e204-8.

39. Turner H, Marshall E, Stopa L, Waller G. Cognitive-behavioural therapy for outpatients with eating disorders: effectiveness for a transdiagnostic group in a routine clinical setting. Behav Res Ther. 2015;68:70-5.

40. Waller G, Gray E, Hinrichsen H, Mountford V, Lawson R, Patient E. Individualized cognitive behavioural therapy for bulimia nervosa and atypical bulimic cases: Generalisability of effectiveness to clinical settings. Int J Eat Disord. 2014;47:13-7.

41. Tobin DL, Banker JD, Weisberg L, Bowers W. I know what you did last summer (and it was not CBT): a factor analytic model of international psychotherapeutic practice in the eating disorders. Int J Eat Disord. 2007;40:754-7.

42. Wallace LM, von Ranson KM. Treatment manuals: use in the treatment of bulimia nervosa. Behav Res Ther. 2011;49:815-20.

43. von Ranson KM, Robinson KE. Who is providing what type of psychotherapy to eating disorder clients? A survey. Int J Eat Disord. 2006;39:27-34.

44. Kosmerly S, Waller G, Robinson AL. Clinician adherence to guidelines in the delivery of family-based therapy for eating disorders. Int J Eat Disord. 2015;48:223-9. 
45. Simmons AM, Milnes SM, Anderson DA. Factors influencing the utilization of empirically supported treatments for eating disorders. Eat Disord. 2008; 16:342-54.

46. von Ranson KM, Wallace LM, Stevenson A. Psychotherapies provided for eating disorders by community clinicians: Infrequent use of evidence-based treatment. Psychother Res. 2013;23:333-43. Shows how uncommon it is for therapists to use evidencebased approaches to psychotherapy for eating disorders.

47. Waller G, Stringer H, Meyer C. What cognitive-behavioral techniques do therapists report using when delivering cognitivebehavioral therapy for the eating disorders? J Consult Clin Psychol. 2012;80:171-5.

48. Cowdrey ND, Waller G. Are we really delivering evidence-based treatments for eating disorders? How eating-disordered patients describe their experience of cognitive behavioral therapy. Behav Res Ther. 2015;75:72-7. Demonstrates that patients' experiences of 'evidence-based' therapies are commonly dissimilar to the protocols for those therapies.

49. Serpell L, Stobie B, Fairburn CG, van Schaick R. Empiricallysupported and non-empirically supported therapies for bulimia nervosa: retrospective patient ratings. J Eating Disorders. 2013;1: 41-9.

50. Murphy R, Straebler S, Cooper Z, Fairburn CG. Cognitive behavioral therapy for eating disorders. Psychiatr Clin N Am. 2010;33: 611-27.

51. Lowe MR, Bunnell DW, Neeren AM, Chernyak Y, Greberman L. Evaluating the real-world effectiveness of cognitive-behavior therapy efficacy research on eating disorders: a case study from a community-based clinical setting. Int J Eat Disord. 2011;44:9-18.

52. Cowdrey ND. Perspectives on eating disorders: Attitudes to sufferers, and patient experiences of what goes on in cognitive behavioural therapy. DClinPsy dissertation, University of Sheffield, Sheffield, UK; 2015.

53. Royal College of Psychiatrists. National Audit of Psychological Therapies for Anxiety and Depression, National Report 2011. London: Healthcare Quality Improvement Partnership; 2011.

54. Royal College of Psychiatrists. Report of the Second Round of the National Audit of Psychological Therapies (NAPT) 2013. London: Healthcare Quality Improvement Partnership; 2013.

55.• Poulsen S, Lunn S, Daniel SI, Folke S, Mathiesen BB, Katznelson $\mathrm{H}$, et al. A randomized controlled trial of psychoanalytic psychotherapy or cognitive-behavioral therapy for bulimia nervosa. Am J Psychiatr. 2014;171:109-16. A very clear demonstration of the difference in effects between CBT and dynamic psychotherapy for bulimia nervosa.

56. Walfish S, McAlister B, O'Donnel P, Lambert M. An investigation of self-assessment bias in mental health providers. Psychol Rep. 2012;110:1-6.

57. Parker Z, Waller G. Factors related to psychotherapists' selfassessment bias when treating anxiety disorders. Behav Res Ther. 2015;66:1-7.

58. Hansen NB, Lambert MJ, Forman EM. The psychology doseresponse effect and its implications for treatment delivery services. Clin Psychol Sci Pract. 2002;9:308-27.

59. Waller G, Mountford VA, Tatham M, Turner H, Gabriel C, Webber R. Attitudes towards psychotherapy manuals among clinicians treating eating disorders. Behav Res Ther. 2013;51:840-4.

60. Dray J, Wade TD. Is the transtheoretical model and motivational interviewing approach applicable to the treatment of eating disorders? A review. Clin Psychol Rev. 2012;32:558-65.

61. Waller G. The myths of motivation: time for a fresh look at some received wisdom in the eating disorders? Int J Eat Disord. 2012;45: $1-16$.

62. Brown A, Mountford VA, Waller G. An idea worth researching: is the therapeutic alliance overvalued in the treatment of the eating disorders? Int J Eat Disord. 2013;46:779-82.
63. Graves TA, Tabri N, Thompson-Brenner H, Franko DL, Eddy K, Bourion-Bedes S, Brown A, Constantino MJ, Flückiger C, Forsberg S, Hildebrandt T, Isserlin L, Couturier J, Karlsson GP, Mander J, Teufel M, Mitchell JE, Crosby RD, Prestano C, Satir DA, Simpson S, Sly R, Lacey JH, Stiles-Shields C, Tasca GA, Waller G, Zaitsoff SL, Rienecke R, Le Grange D, Thomas JJ. (under consideration). A meta-analysis of the relation between therapeutic alliance and treatment outcome in eating disorders. Clin Psychol Rev.

64. Waller G, Mountford VA. Weighing patients within cognitivebehavioural therapy for eating disorders: how, when and why. Behav Res Ther. 2015;70:1-10.

65. Wonderlich S, Mitchell JE, Crosby RD, Myers TC, Kadlec K, Lahaise K, et al. Minimizing and treating chronicity in the eating disorders: a clinical overview. Int J Eat Disord. 2012;45. 467-75.

66. Karačić M, Wales JA, Arcelus J, Palmer RL, Cooper Z, Fairburn CG. Changes in alcohol intake in response to transdiagnostic cognitive behaviour therapy for eating disorders. Behav Res Ther. 2011;49:573-7.

67. Thompson-Brenner H, Westen D. Personality subtypes in eating disorders: validation of a classification in a naturalistic sample. $\mathrm{Br}$ J Psychiatry. 2005;186:516-24.

68. Meyer JM, Farrell NR, Kemp JJ, Blakey SM, Deacon BJ. Why do clinicians exclude anxious clients from exposure therapy? Behav Res Ther. 2014;54:49-53. Clear evidence that clinicians' own characteristics influence the implementation of evidence-based therapies.

69. Meehl PE. Why I do not attend case conferences. In: Meehl PE, editor. Psychodiagnosis: Selected papers (pp. 225-302). Minneapolis: University of Minnesota Press; 1973.

70. Institute of Medicine. Crossing the quality chasm: a new health system for the 21st century. Washington: National Academy Press; 2001.

71. Fairburn CG, Cooper Z. Therapist competence, therapy quality, and therapist training. Behav Res Ther. 2011;49:373-8.

72. Deacon BJ, Farrell NR, Kemp JJ, Dixon LJ, Sy JT, Zhang AR, et al. Assessing therapist reservations about exposure therapy for anxiety disorders: the therapist beliefs about exposure scale. J Anxiety Disorders. 2013;27:772-80.

73. Waller G, D'Souza K, Wright C. Impact of education on clinicians' attitudes to exposure therapy for eating disorders. Behav Res Ther. 2016;76:76-80.

74. Dennhag I, Gibbons MB, Barber JP, Gallop R, Crits-Christoph P. Do supervisors and independent judges agree on evaluations of therapist adherence and competence in the treatment of cocaine dependence? Psychother Res. 2012;22:720-30.

75. De Jong K, van Sluis P, Nugter MA, Heiser WJ, Spinhoven P. Understanding the differential impact of outcome monitoring: therapist variables that moderate feedback effects in a randomized clinical trial. Psychother Res. 2012;22:464-74.

76. Meehl PE. Causes and effects of my disturbing little book. J Pers Assess. 1986;50:370-5.

77. Wilson GT, Loeb KL, Walsh BT, Labouvie E, Petkova E, Lui X, et al. Psychological versus pharmacological treatments of bulimia nervosa: predictors and processes of change. J Consult Clin Psychol. 1999;67:451-9.

78. Agras WS, Crow SJ, Halmi KA, Mitchell JE, Wilson GT, Kraemer HC. Outcome predictors for the cognitive behavior treatment of bulimia nervosa: data from a multisite study. Am J Psychiatr. 2000;157:1302-8.

79.• Raykos BC, Watson HJ, Fursland A, Byrne SM, Nathan P. Prognostic value of rapid response to enhanced cognitive behavioral therapy in a routine clinic sample of eating disorder outpatients. Int J Eat Disord. 2013;46:764-70. Confirming the importance of early change in treating eating disorders. 
80.• Turner H, Bryant-Waugh R, Marshall E. The impact of early symptom change and therapeutic alliance on treatment outcome in cognitive-behavioural therapy for eating disorders. Behav Res Ther. 2015;73:165-9. Demonstrating the superiority of early change as a predictor of outcomes, relative to the presumed role of the working alliance.
81. Keel PK, Dorer DJ, Franko DL, Jackson SC, Herzog DB. Postremission predictors of relapse in women with eating disorders. Am J Psychiatr. 2005;162:2263-8.

82. Turner H, Tatham M, Lant M, Mountford VA, Waller G. Clinicians' concerns about delivering cognitive-behavioural therapy for eating disorders. Behav Res Ther. 2014;57:38-42. 